105

\section{USING CARDIAC MAGNETIC RESONANCE TO PREDICT CLINICAL OUTCOMES IN EISENMENGER SYNDROME}

S J Fitzsimmons, H Nashat, A J Carroll, S Thomas, C Peebles, G R Veldtman

University Hospital Southampton

\section{doi:10.1136/heartjnl-2013-304019.105}

Introduction Eisenmenger Syndrome is defined as pulmonary hypertension with reversed or bi-directional shunting at any level between the systemic and pulmonary circulations. Retrospective studies have identified predictors of clinical outcomes including: age, function class and resting oxygen saturations. Cardiac magnetic resonance (CMR) imaging provides direct evaluation of subpulmonary ventricular function, morphology, stroke volume and myocardial mass, whilst allowing a non-invasive method of shunt calculation. For these reasons CMR is an attractive modality for predicting outcomes in Eisenmenger Syndrome.

Aim To assess whether Cardiac Magnetic Resonance (CMR) measurements of the sub-pulmonary ventricle can predict clinical outcomes in Eisenmenger Syndrome.

Methods 19 patients, aged 18 years or over with the diagnosis of Eisenmenger Syndrome on transthoracic echocardiography and/or catheter catheterisation were recruited between 2008 and 2011. Each patient underwent a hospital standardised CMR protocol with an ECG gated CMR (1.5 Tesla MR). Ventricular systolic and diastolic volumes, myocardial mass, ejection fraction and $\mathrm{Op}$ : Os were calculated by a single operator. Baseline demographics and predictors of clinical outcomes were recorded including: resting oxygen saturations, 6-min walk distance (6 MWD) and NYHA functional class.

Mean values are shown \pm SDs for normally distributed continuous variables. Non-parameter testing used Spearman's rank correlation.

Results 15 patients were female (79\%). Group mean age was 34.8 \pm 11 years (age range $18-52$ years). 6 patients (31\%) within our population had Down Syndrome. Diagnoses included; complete $\operatorname{AVSD}(n=4), \operatorname{VSD}(n=5), \operatorname{PDA}(n=3)$, secundum ASD $(n=2)$, complex $(n=5)$. Mean sub-pulmonary ventricular (SPV) ejection fraction was $52 \% \pm 13$, systolic volumes $44.7 \mathrm{ml} / \mathrm{m}^{2} \pm 26$, diastolic volumes $94.9 \mathrm{ml} / \mathrm{m}^{2} \pm 45$, stroke volume $48.6 \mathrm{ml} / \mathrm{m}^{2} \pm 33$, myocardial mass $66.3 \mathrm{~g} / \mathrm{m}^{2} \pm 23$ and cardiac output $6.2 \mathrm{l} / \mathrm{min} \pm 4$. Mean systemic ventricular ejection fraction was $58 \% \pm 9.2$, systolic volumes $37.7 \mathrm{ml} / \mathrm{m}^{2} \pm 28$, diastolic volumes $63.6 \mathrm{ml} / \mathrm{m}^{2} \pm 44$, stroke volume $37.3 \mathrm{ml} / \mathrm{m}^{2} \pm 28$, myocardial mass $51.6 \mathrm{~g} / \mathrm{m}^{2} \pm 22$ and cardiac output $4.4 \mathrm{~L} / \mathrm{min} \pm 4$.

$6 \mathrm{MWD}$ was highly correlated with; resting saturations $(\mathrm{R}=0.61$ : $p=0.01)$, functional class $(R=-0.53: p=0.03)$, sub-pulmonary ventricular myocardial mass $(R=0.82$ : $p=0.002)$, sub-pulmonary ventricular systolic volume $(R=0.85: p=0.001)$ and sub-pulmonary ventricular diastolic volume $(R=0.07: p=0.01)$. Resting saturations were highly correlated with: Age $(R=0.56: p=0.008)$, functional class $(R=-0.66: p=0.001)$.

Conclusions CMR derived SPV myocardial mass and volumes correlated with functional clinical outcomes within our cohort. $6 \mathrm{MWD}$ increased with greater SPV myocardial mass, consistent with favourable SPV remodelling. CMR offers further knowledge into adaptations of the subpulmonary ventricle and may produce additional information to current predictors of outcomes in Eisenmenger Syndrome. 